\title{
MR Imaging of the Adnexal Masses: A Review
}

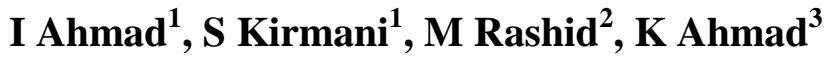 \\ ${ }^{1}$ Department of Radiodiagnosis, J.N. Medical College and Hospital, A.M.U, Aligarh, U.P; \\ India. ${ }^{2}$ Department of Medical Imaging, Western Health, Melbourne, VIC, Australia. \\ ${ }^{3}$ Department of Radiodiagnosis, B.P. Koirala Institute of Medical Sciences, Dharan, Nepal.
}

\begin{abstract}
MR (magnetic resonance) imaging is a non invasive technique for evaluation of female pelvic masses. Due to its high spatial resolution and excellent tissue contrast, various masses of adnexal origin can be imaged and a confident diagnosis can be made. MRI helps to delineate normal anatomical structures and elucidate the pathological lesions. It has high sensitivity and specificity for differentiating benign adnexal masses from malignant ones. This review article gives a brief account of approach to adnexal masses based on tissue characterization on MR imaging.
\end{abstract}

Keywords: MRI, Adnexa, Malignancy, Ovarian tumor

\section{Introduction}

Magnetic resonance (MR) imaging is a valuable modality for non invasive evaluation of the female pelvic region. It has become an important tool in diagnosing benign and malignant masses of the female pelvis. MRI is an excellent modality, which helps to delineate the normal anatomical structures and elucidate the nature and origin of variety of pathological conditions in female pelvis. MR Imaging is being increasingly used to evaluate adnexal masses because of its excellent spatial and tissue contrast resolution, good tissue characterization and multiplanar imaging capability. Moreover, MRI does not depend on ionizing radiations.

Correspondence to: Dr. Sanna Kirmani Department of Radiodiagnosis JNMCH, AMU, Aligarh UP, India, 202002, E-mail:sannakirmani@gmail.com

\section{Why MRI?}

USG (ultrasonography) is considered as first line investigation for a suspected adnexal mass because of its wide availability and relatively low cost. However there are some shortcomings with this imaging modality such as limited field of view, obscuration of pelvic organs by presence of bowel gas, dependence on patients' size and experience of the operator. Further number of these adnexal masses may be "indeterminate" on sonography. John et $\mathrm{al}^{1}$ has defined a sonographically indeterminate adnexal mass as (a) one that has complexity \& cannot be confidently placed into either the benign or malignant category or (b) one for which the site of origin, from the ovary, uterus, or another pelvic structure, remains to be established. MRI has a high diagnostic accuracy for benign and malignant adnexal masses. In a study of 91 benign and 96 malignant adnexal masses, gadoliniumenhanced MRI depicted $94 \%$ of adnexal 
masses and had an overall accuracy of 93\% for diagnosis of malignancy ${ }^{2}$.

\section{MR Protocol}

Before imaging patient should fast for 6 hours to decrease peristalsis or alternatively glucagon may be administered prior to examination. Patient should void before examination to prevent deformity of adjacent organs and avoid a restless patient due to over distended bladder. A pelvic phased array coil or body coil is used to increase signal-tonoise ratio. T1 weighted axial images are obtained using spin echo technique and T2 weighted images are obtained in sagittal, axial and coronal planes using fast spin echo technique. Additional sequences are tailored depending upon the abnormal adnexal features on initial imaging sequences. Post contrast images are obtained in suspected malignant masses. Fat suppression sequences are required for characterizing bright lesion on T1-weighted images (such as fat vs. blood). Chemical shift imaging also helps differentiate fat from blood.

\section{MRI Criteria for Differentiating Benign and Malignant Adnexal Masses:}

MRI has a high diagnostic accuracy for differentiation of benign from malignant masses of the adnexa and this improves with the use of gadolinium based contrast agents. Komatsu et $\mathrm{al}^{3}$ found that the single criteria of enhancing solid tissue was $91 \%$ sensitive and $88 \%$ specific for differentiation of benign from malignant adnexal masses. The MRI features most predictive of malignancy are necrosis in a solid lesion and vegetations in a cystic lesion ${ }^{2}$. Features that suggest either benign or malignant epithelial neoplasms include:

\section{Tissue Characterization with MR Imaging}

On the basis of the signal intensity, adnexal masses may be placed in one of the following four categories:

\section{High T1 Signal:}

These include lesions containing either fat or hemorrhagic products such as dermoids/ teratomas, hemorrhagic cysts, endometriomas and cyst with proteinaceous and mucinous contents and rarely metastatic melanoma. Fat suppression sequences will help to narrow down the differentials, as fat containing dermoids and teratoma will be dark on these sequences (Fig 1 a \& b) and blood containing lesions will remain bright even after fat suppression. Further, hemorrhagic lesion such as endometriomas show T2 shading sign i.e., a hypointense rim and internal shading (a peripheral area of low signal intensity) that is most prominent on $\mathrm{T} 2$ weighted images (Fig 2 a, b, c). Also these hemorrhagic lesions may show dark-bright fluid

levels.

\section{High T2, Low T1 Signal:}

These include simple cysts, cystadenomas and hydrosalpinx. Simple cysts are thin walled, have homogenous signal intensity. Serous cystadenomas are usually unilocular and may have thin septations $(<3 \mathrm{~mm})$ and few papillary projections. Mucinous cystadenomas are multiloculated and have bright signal on $\mathrm{T} 1$ owing to their mucin content. Hydrosalpinx appears as a convoluted, elongated structure with incomplete folds or plica (Fig 3).

\section{Low T1, Low T2 Signal:}

An adnexal mass that is low in signal intensity on T1- and T2-weighted images is most likely a pedunculated leiomyoma ${ }^{4}$. Ovarian fibromas also present with low signal intensity on $\mathrm{T} 1$ - and $\mathrm{T} 2$-weighted sequences. With an ovarian fibroma there may be a small amount of normal remnant ovary draped around the solid mass or the solid mass may be exophytic from the ovarian tissue ${ }^{5}$. 


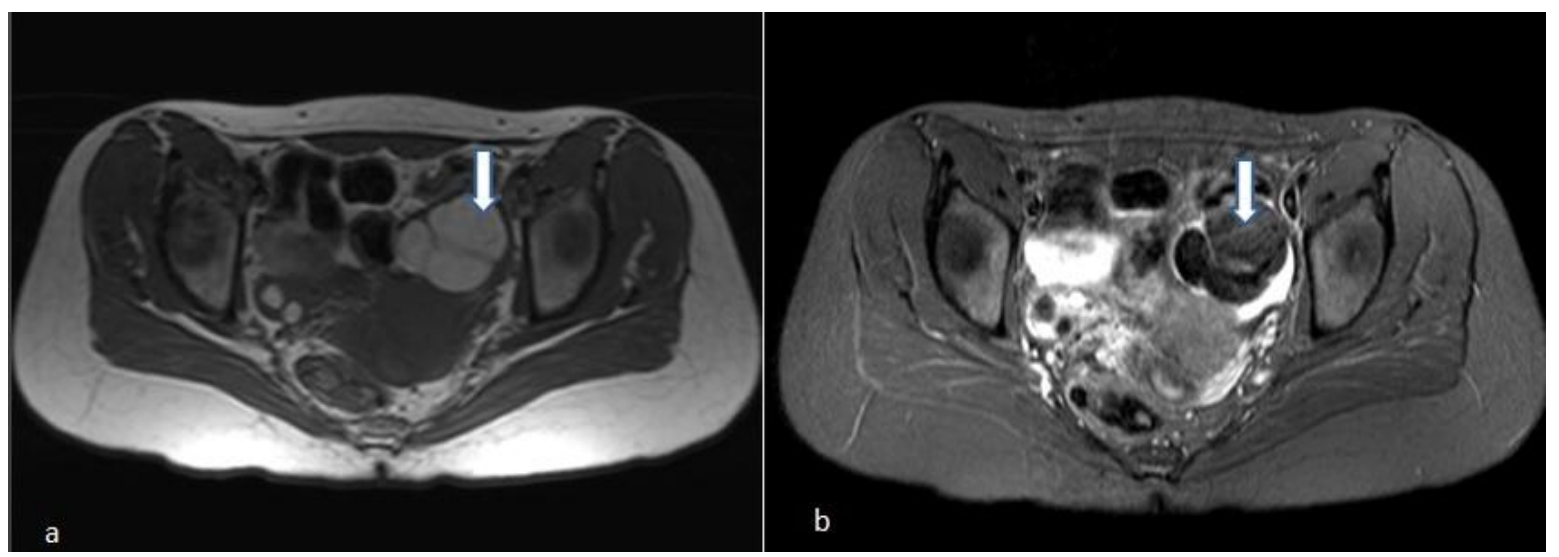

Figure 1: Dermoid : Axial T1 weighted MR image(a) showing a "bright" adnexal lesion on left side (arrow) with loss of this bright signal on fat suppressed post contrast T1weighted image(b), confirming the presence of fat.
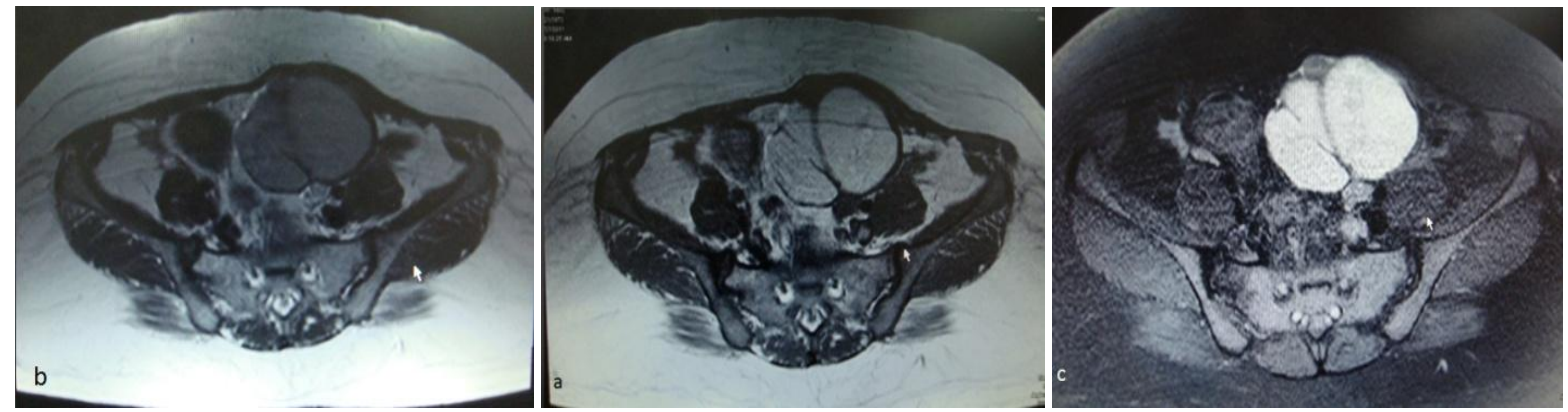

Figure 2: Endometrioma: Axial T1-weighted MR image (a) showing a "bright" adnexal lesion on left side which appears hypointense on T2-weighted image (b) with a well defined hypointense rim (T2-shading). The lesion remains bright even on fat suppressed proton density sequence $(c)$.

\section{Heterogenous Signal}

These include primarily malignant masses, structure on T2-weighted images. The cyst is lesions with haemorrhage of various ages, inflammatory masses, and complications of any of the above mentioned masses, such as torsion of normal ovary or an ovarian mass. Contrast enhanced MR scans play an important role in differentiation of these masses.

\section{Pathology:}

\section{Simple Cyst:}

A simple adnexal cyst appears as a homogenous low signal intensity structure on T1 weighted images and high signal intensity well circumscribed with imperceptible wall and has no solid component (Fig 4).

\section{Endometriomas:}

Endometrial cysts vary in size from millimetres to many centimetres. Multiplicity of lesions and adhesions between lesions and other adjacent structures is characteristic. MR imaging can depict the presence of blood products in these lesions. These lesions usually appear bright on T1W images and low signal intensity on T2W images (Fig 2); however their appearance may be variable and heterogeneous depending upon the age of the haemorrhage. Low signal on T2-weighted 


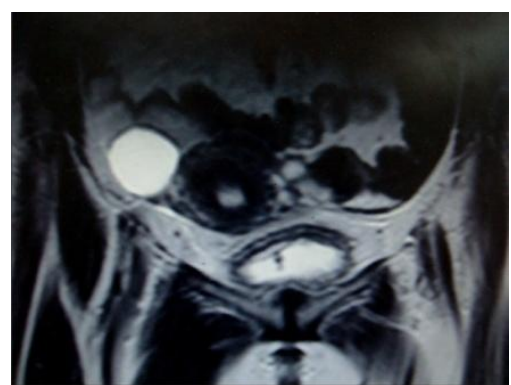

Figure 3: Hydrosalpinx: Coronal T2-weighted MR image showing dilated convoluted fallopian tubes bilaterally.

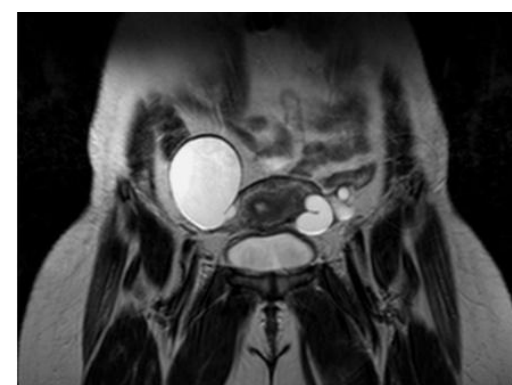

Figure4: Simple Coronal T2-weighted MR image showing hyperintense lesion with thin wall and no solid component in right adnexa.

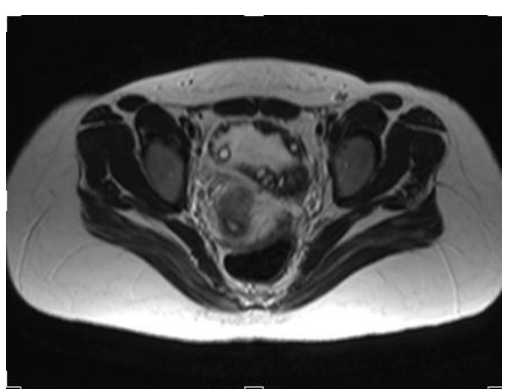

Figure 5: Ovarian Torsion: Axial T2-weighted MR image showing enlarged left ovary with peripherally arranged follicles and edematous stroma
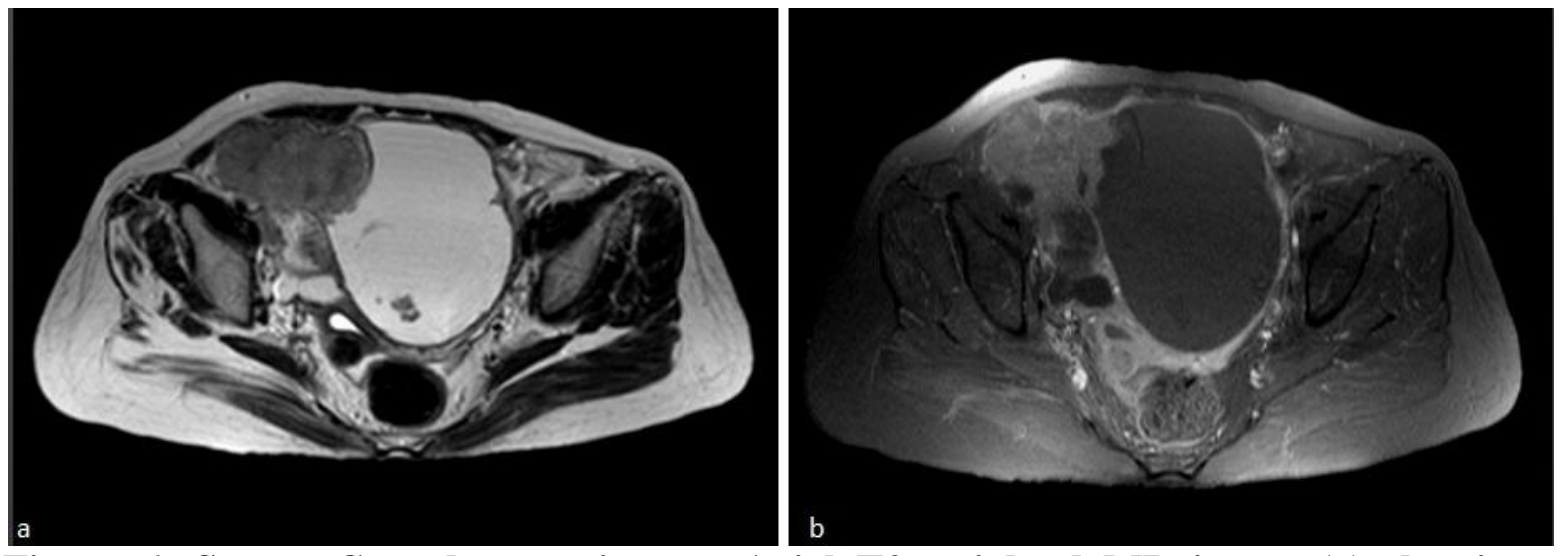

Figure 6: Serous Cystadenocarcinoma: Axial T2 weighted MR image (a) showing a complex solid cystic lesion in left adnexa with infiltration of adjacent pelvic organs. Axial fat suppressed post contrast T1-weighetd MR image (b) showing enhancement of the solid component of the lesion and its wall.

images is due to presence of iron in these lesions. Multiplicity, bilateralism and lesions in cul de sac in an appropriate clinical scenario, is diagnostic of endometriosis.

\section{Hydrosalpinx:}

It may appear as a purely cystic or a complex cystic mass in the adnexa. Heterogeneous signal intensity within it may be due to pus, debris and other inflammatory contents. It has a characteristic serpiginous or sausage like appearance with incomplete folds (septations) within it (Fig 3). Short linear projections, also known as cog-wheel sign may be appreciated on T2-weighted images. Adjacent edema and

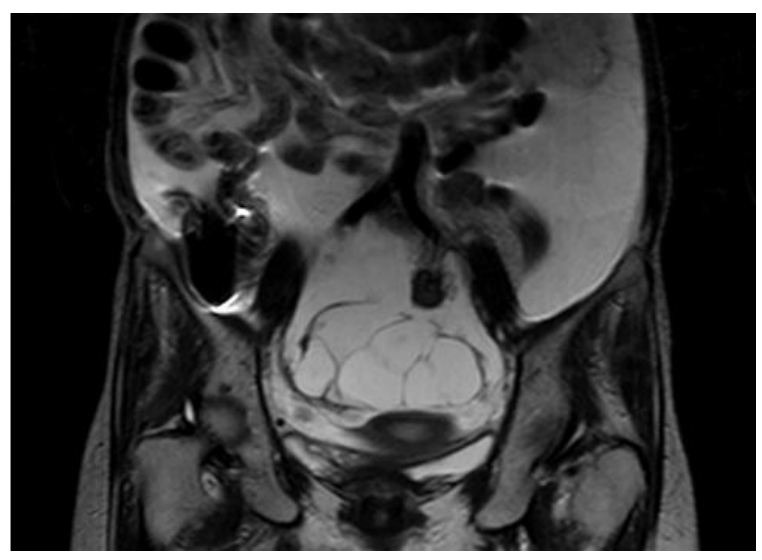

Figure 7: Mucinous Cystadenocarcinoma: Coronal T2-weighted MR image showing a multilocular cystic mass arising from the pelvis with gross ascites. 

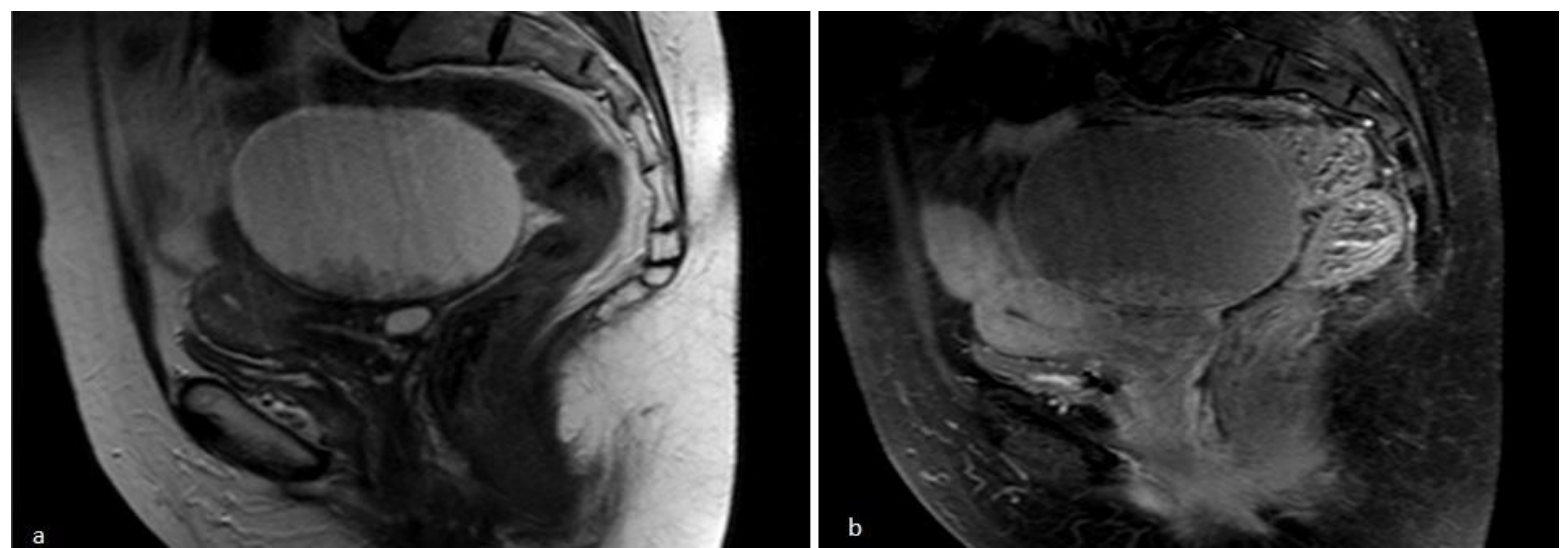

Figure 8: Endometrioid Tumour: Sagittal T2-weighted (a) and post contrast fat suppressed T1-weighted MR image showing a cystic lesion with enhancing solid component within it, which at histopathology proved to be an endometrioid tumour.
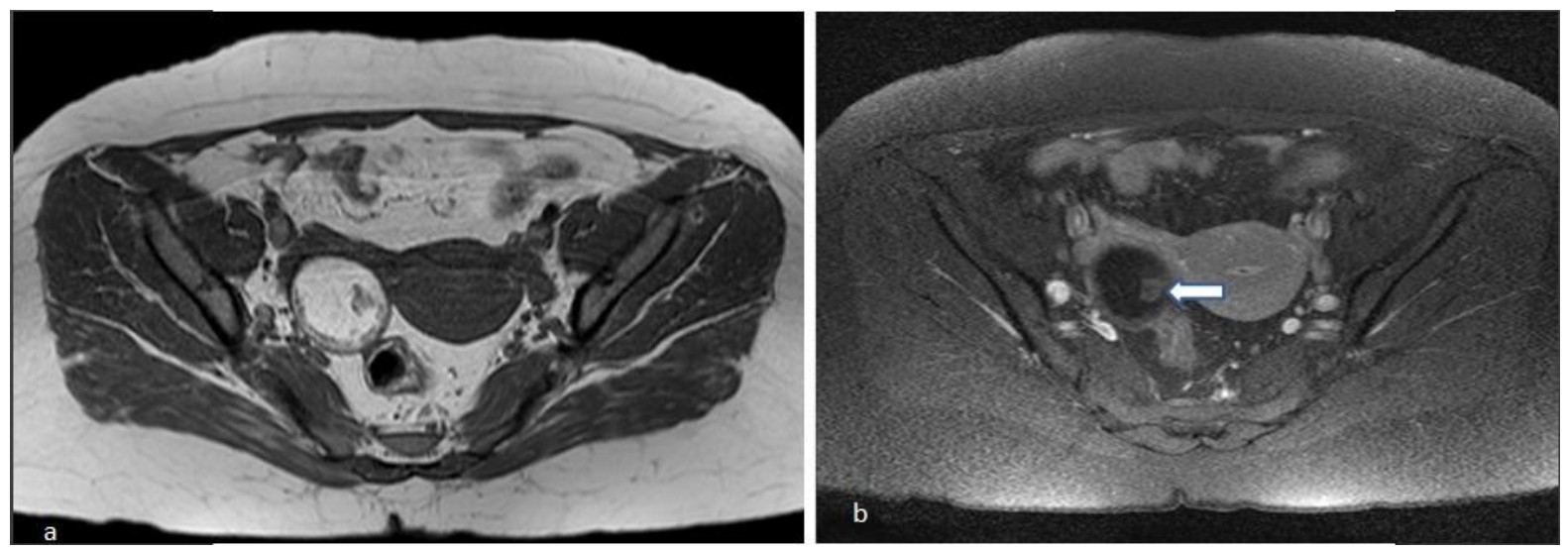

Figure 9: Immature Teratoma: A bright lesion is seen on axial T1-weighted MR image (a) with a hypointense nodule within it. Loss of this bright signal is seen on post contrast fat suppressed T1-weighted MR image (b), suggestive of fat content, with enhancement of the nodule. Histopathology proved an immature teratoma.

tendency to adhere to adjacent tissue may be appreciated.

\section{Ovarian Torsion:}

At an early stage, a torsed ovary is swollen with multiple follicles separated by edematous stroma (Fig 5). A common finding in ovarian torsion is the presence of a thick pedicle between the mass and the uterus. MR diagnosis of ovarian torsion is based on three cardinal features as described by Kimura et $\mathrm{al}^{6}$ (a) engorged blood vessels on the side of torsion with a protrusion of the lesion toward the uterus, (b) lack of enhancement, and (c) straight blood vessels draped around the lesion. Haemorrhage within a torsed ovary may result in high signal on T1-weighted images and subsequent necrosis a high signal on

T2-weighted

images.

\section{Ovarian Tumors:}

MRI has been shown to have a 91-93\% overall accuracy for differentiating benign from malignant adnexal tumors ${ }^{7}$. MRI also increases the specificity of diagnosis in suspicious masses on TVUS (Transvaginal ultrasonographically). MR imaging was most valuable when the tumor marker CA-125 level was normal or only minimally elevated ${ }^{8}$.

Although MRI can differentiate between 
benign, borderline and malignant epithelial lesions, specific histological differentiation Table- 1

\begin{tabular}{lll}
\hline Variable & Benign & Malignant \\
\hline Size & Usually less than $4 \mathrm{~cm}$ & Usually more than $4 \mathrm{~cm}$ \\
Component & Predominantly cystic & Predominantly solid or solid-cystic \\
Wall thickness & Thin $(<3 \mathrm{~mm})$ & Thick $(>3 \mathrm{~mm})$ \\
Septa & Thin $(<3 \mathrm{~mm})$ & Thick $(>3 \mathrm{~mm})$ \\
Internal structure & Lacking & $\begin{array}{l}\text { Papillary projections, vegetations, } \\
\text { nodularity }\end{array}$ \\
Necrosis & Absent & Cardinal feature \\
Ascites & None & Peritoneal, anterior to uterus \\
Other & ------ & $\begin{array}{l}\text { Peritoneal implants, pelvic wall } \\
\text { invasion, adenopathy, } \\
\end{array}$ \\
& & Distant metastases \\
\hline
\end{tabular}

cannot be made. Serous epithelial tumours usually appear hypointense on T1-weighted images (Fig 6) and mucinous tumours appear hyperintense due to high mucin content aiding in differentiation between the two. While serous tumours are unilocular and bilateral, mucinous tumours are multilocular and unilateral (Fig 7). Papillary tumours are distinctive features of epithelial tumours.

Endometrioid tumour is large, complex cystic mass with solid components (Fig 8). In about $15 \%-30 \%$ it is associated with synchronous endometrial carcinoma or endometrial hyperplasia. ${ }^{9}$ Brenner tumour usually presents as a solid mass or a complex solidcystic lesion. Brenner tumour is composed of transitional cells with fibrous stroma which gives it low signal intensity on T2 weighted images. Calcifications may be seen in Brenner's tumour. $^{10}$

The most common germ cell tumour is a mature teratoma. Mature teratoma is the most common benign ovarian tumor in women less than 45 years old. These tumours are characterized by presence of fat, bone, teeth, hair follicles and muscle. Because of presence of fat, high signal intensity on T1weighted images with loss of this signal on fat suppressed sequences are characteristic.
Immature or malignant teratomas have prominent solid components and may demonstrate internal necrosis or haemorrhage (Fig 9). Dysgerminoma appears as solid masses with enhancing fibrovascular septae. Granulosa cell tumors are estrogen producing and have a variable appearance on imaging ranging from solid, cystic or hemorrhagic.

Krukenberg tumors are metastatic tumors to the ovary that contain mucin-secreting "signet ring" cells. These usually originate in the gastrointestinal tract. ${ }^{11}$ Metastatic lesions more commonly bilateral, with maintained oval shape of the ovary, and contain welldemarcated intratumoral cysts with strongly enhancing walls. ${ }^{12}$ Metastatic lesions appear hypointense on T2-weighted images due to dense stroma and hyperintense on T1weighted images due to presence of mucin. A prominent MR imaging sign of a Krukenberg tumor is the presence of large intralesional radiating flow voids.

\section{Conclusion}

MR is the state of the art imaging modality for adnexal masses with a high overall diagnostic accuracy. Tissue characterization based on differences in MR relaxation properties seen in various types of tissues, is 
invaluable in diagnosing adnexal masses. MR also aids in staging of malignant adnexal masses. Gadolinium-enhanced MR imaging serves as a problem-solving modality in cases of indeterminate adnexal masses.

\section{References}

1. John A. Spencer, Sunethra Ghattamaneni. MR Imaging of the Sonographically Indeterminate Adnexal Mass. Radiology 2010; 256(3):677-694.

2. Hricak H, Chen M, Coakley FV, et al. Complex adnexal masses: detection and characterization with MRI-multivariate analysis. Radiology 2000; 214:39-46.

3. Komatsu T, Konishi I, Mandai M, et al. Adnexal masses: transvaginal US and gadolinium-enhanced MRI assessment of intratumoral structure. Radiology 1996; 198:109-115.

4. Weinreb JC, Barkoff ND, Megibow A, et al. The value of MRI in distinguishing leiomyomas from other solid pelvic masses when sonography is indeterminate. AJR Am J Roentgenol 1990; 154:295-299.

5. Madan R. The bridging vascular sign. Radiology 2006; 238 (1): 371-372.
6. Kimura I, Togashi K, Kawakami S, et al. Ovarian torsion: CT and MRI appearances. Radiology 1994; 190:33741.

7. Sohaib SA, Sahdev A, Van Trappen P, et al. Characterization of adnexal lesions on MR imaging. AJR 2003; 180:1297-1304.

8. Sohaib SA, Mills TD, Sahdev A, et al. The role of magnetic resonance imaging and ultrasound in patients with adnexal masses. Clin Radiol 2005; 60(3):340-348

9. Buy JN, GhossainMA, Sciot C, et al. Epithelial tumors of the ovary: CT findings and correlation with US. Radiology 1991; 178:811 -818.

10. Moon WJ, Koh BH, Kim SK, et al. Brenner tumor of the ovary: CT andMR findings. J Comput Assist Tomogr 2000; 24:72-76

11. Ha HK, Baek SY, Kim SH, et al. Krukenberg's tumor of the ovary: MR imaging features. AJR Am J Roentgenol 1995;164:1435-1439.

12. Kim SH, Kim WH, Park KJ, et al. CT and MR findings of Krukenberg tumors: comparison with primary ovarian tumors. J Comput Assist Tomogr 1996; 20:393398. 